\title{
Capillary Filling in Closed End Nanochannels
}

\author{
Vinh Nguyen Phan, ${ }^{\dagger}$ Nam-Trung Nguyen, ${ }^{* \dagger}$ Chun Yang, ${ }^{\dagger}$ Pierre Joseph, ${ }^{\dagger}$ Lyes \\ Djeghlaf, ${ }^{\ddagger}$ David Bourrier ${ }^{\ddagger}$ and Anne-Marie Gue ${ }^{\ddagger}$
}

School of Mechanical and Aerospace Engineering, Nanyang Technological University, 50 Nanyang Avenue, Singapore, Singapore 639798, and CNRS, LAAS, Université de Toulouse, 7 Avenue du Colonel Roche, Toulouse, France F-31077

E-mail: mntnguyen@ntu.edu.sg

\begin{abstract}
We investigated the interactions between liquid, gas and solid phases in the capillary filling process of closed-end nanochannels. This paper presents theoretical models without and with absorption and diffusion of gas molecules in the liquid. Capillary filling experiments were carried out in closed-end silicon nanochannels with different lengths. The theoretical and measured characteristics of filling length versus time are compared. The results show that the filling process consists of two stages. The first stage resembles the capillary filling process in an open-end nanochannel. However, a remarkable discrepancy between the experimental results and the theory without gas absorption is observed in the second stage. A closer investigation of the second stage reveals that the dissolution of gas in the liquid is important and can be explained by the model with gas absorption and diffusion.
\end{abstract}

\footnotetext{
*To whom correspondence should be addressed

${ }^{\dagger}$ School of Mechanical and Aerospace Engineering, Nanyang Technological University, 50 Nanyang Avenue, Singapore, Singapore 639798

†CNRS, LAAS, Université de Toulouse, 7 Avenue du Colonel Roche, Toulouse, France F-31077
} 


\section{Introductions}

Interactions among gas, liquid and solid phases in nanoscale play an significant role for many industrial processes. ${ }^{1,2}$ In the past, most of the investigations on nanoscale transport phenomena were carried out with porous materials. $\mathrm{Shaw}^{3}$ reported experimental results of the evaporation process in porous materials. This work set a foundation for subsequent works on modeling of the drying process in porous materials. Prat ${ }^{4}$ used invasion percolation theory to model the drying processes in porous materials. The model was implemented numerically. Yiotis et al. ${ }^{5}$ presented a theoretical model on the drying process in porous materials. This model shows that the formation of a liquid film accelerates the drying process. The model was confirmed experimentally. Recent advances in nanotechnology allows the investigation of liquid transport in deterministic structures such as nanochannels. Experimental results showed discrepancy from what is expected in the larger scale. Eijkel et al. ${ }^{6}$ showed that film flow and corner flow in nanochannel increase the drying speed of liquid.

Capillary filling experiments in open-end channel were conducted previously by many authors. ${ }^{7-10}$ Most of them reported a lower filling speed as predicted by classical theory. This reduction is explained by different hypotheses. Tas et al. ${ }^{7}$ addressed the issue by electro-viscous effect. However, the authors have excluded the effect of bubble formation by selecting a filling range, in which no large bubble formation was observed. Thamdrup et al. ${ }^{8}$ observed that the formation of air bubbles further reduces the filling speed. The authors also introduce a correlation between the bubble density and the filling rate. Han et al. ${ }^{9}$ performed capillary filling in nanochannels with ethanol, isopropanol, water and mixtures of ethanol and water. The authors explain the reduction in filling rate by the change of dynamic contact angle. Bubble formation is also observed in the case of water filling. Phan et al. ${ }^{10}$ reported an analytical model for the electroviscous effect in nanochannels. A comparison between this analytical result and experimental results from other authors revealed that the electroviscous effect is not the only cause of the reduction in filling speed.

Pesse et al. ${ }^{11}$ investigated the capillary filling process in closed-end microchannels. The authors performed filling experiments in rectangular-cross-section, closed-end microchannels with 
lengths ranging from 50 to $150 \mu \mathrm{m}$. Experiments were carried out with deionized (DI) water. Since the first stage of the capillary filling process is relatively fast in microchannels, the authors mainly reported the results of the second stage where the trapped air dissolves in water. The time scale of the experiments are several hours to a day. Due to the existing water vapor in air and its condensation under high Laplace pressure, the filling process does not have a clean meniscus. The experimental data were compared with a simple theory. However, due to complicated appearance of the meniscus and droplet formation in the trapped air region, the experimental data could not fully fit to theoretical model.

The present paper highlights the importance of gas absorption into liquid. This phenomenon was neglected in all previously reported models of liquid transport in nanochannels. We experimentally investigated the capillary filling process of closed-end nanochannels. In contrast to closed-end microchannels, ${ }^{11}$ the nanoscale channel height allows a relatively slow filling process so that both stages of the filling process can be investigated in details. In our investigation, quantitative measurements of filling lengths versus time were obtained for ethanol and isopropanol to avoid the problems of water in closed-end microchannels as reported previously by Pesse et al. ${ }^{11}$ Experiments were also carried out with DI water to confirm that the same behaviour also exists in nanochannels. The behavior of menisci during the filling process and the formation of air bubbles were recorded and qualitatively investigated. A simple model is developed based on the absorption and diffusion of gas in liquid to predict the position of the meniscus. In the model suggested by Pesse et al. ${ }^{11}$ only absorption and diffusion of air in liquid was considered, due to relatively low Laplace pressure in microchannels compared to atmospheric pressure. In nanochannels, the Laplace pressure is high enough to cause significant air compression. Therefore, air compression plays an important role in the final modeling result. Moreover, while the model used by Pesse et al. assumed the diffusion as in semi-infinite medium, ${ }^{11}$ our model considers the variation of filling length in diffusion process. Comparisons between experimental and theoretical results are made where the meniscus in the experimental data is well defined. 


\section{Theories}

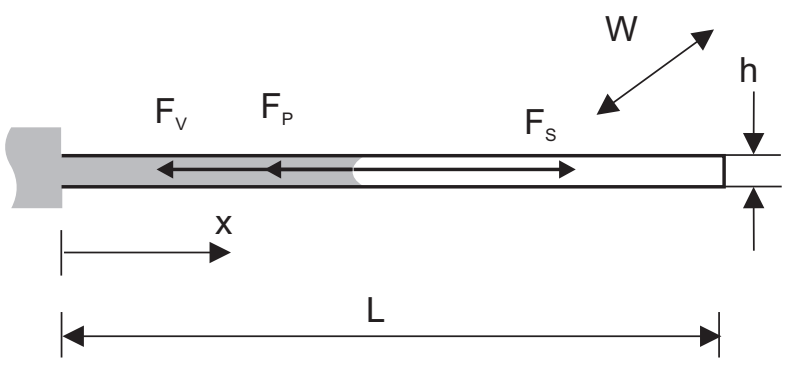

(a)

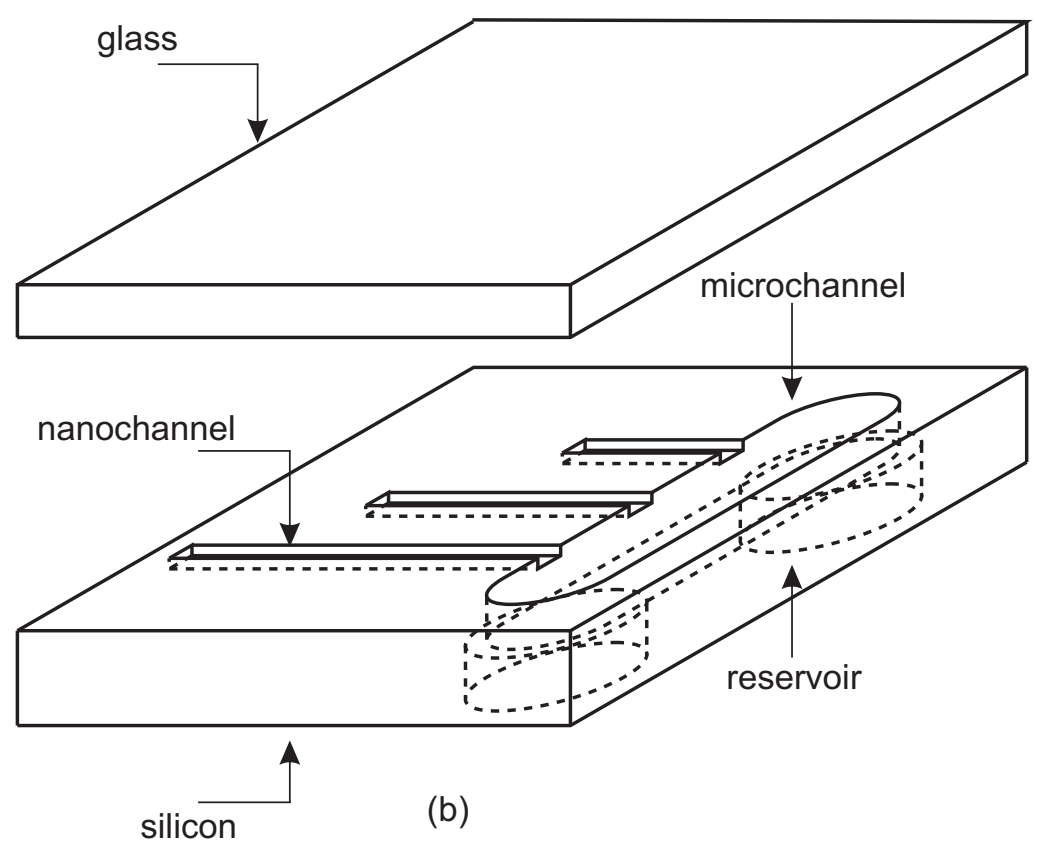

Figure 1: Schematic design of the silicon chips with nanochannels.

The filling process of a closed-end nanochannel depicted in Figure 1(a) is considered. Three forces act on the liquid in the nanochannels. The force $F_{\mathrm{S}}$ caused by the surface tension is the driving force of the whole filling process. The viscous force $F_{\mathrm{V}}$ slows down the filling speed, while the force $F_{\mathrm{P}}$ caused by the pressurized gas trapped in the nanochannels acts against the filling process. Inertia force is negligible in nanoscale. The force balance leads to:

$$
F_{\mathrm{S}}+F_{\mathrm{V}}+F_{\mathrm{P}}=0
$$

The forces in a closed-end planar nanochannel of a height $h$, a width $w(h<<w)$ and a length $L$ 
are:

$$
\left\{\begin{array}{l}
F_{\mathrm{S}}=2 w \sigma \cos \theta \\
F_{\mathrm{V}}=-\frac{12 \mu u}{h} w x \\
F_{\mathrm{P}}=-h w\left(p-p_{0}\right)
\end{array}\right.
$$

where $\sigma$ is the surface tension of the fluid, $\theta$ is the contact angle between the fluid and the wall of the nanochannels, $\mu$ is the dynamic viscosity of the fluid, $u$ is the filling speed, $x$ is the filling length, $p$ is the pressure of the trapped air, $p_{0}$ is the initial pressure of the trapped gas volume. If the experiment is carried out in the atmosphere, $p_{0}$ is the atmospheric pressure. The formulae above are valid with the assumptions that the system is isothermal and the filling velocity profile is parabolic across the channels height.

With negligible gas absorption, the mass of gas trapped in the channels remains constant. The pressure force $F_{\mathrm{P}}$ is determined as:

$$
F_{\mathrm{P}}=-h w p_{0}\left[\frac{L}{L-x}-1\right]=-h w p_{0} \frac{x}{L-x}
$$

The force balance of Eq. (1) leads to

$$
\frac{\mathrm{d} x}{\mathrm{~d} t}=\frac{h \sigma \cos \theta}{6 \mu x}-\frac{h^{2} p_{0}}{12 \mu(L-x)}
$$

Normalizing the length scale by the channel length, the time scale by twice the time needed to fill an open-end channel of the same length $\tau_{\text {filling }}=3 \mu L^{2} /(h \sigma \cos \theta)$, and the pressure by the Laplace pressure leads dimensionless variables $x^{*}=x / L, t^{*}=t / 2 \tau_{\text {filling }}$ and $\alpha=h p_{0} /(2 \sigma \cos \theta)$. The parameter $\alpha$ represents the ratio between the atmospheric pressure and the Laplace pressure. The dimensionless form of Eq. (4) is:

$$
\frac{\mathrm{d} x^{*}}{\mathrm{~d} t^{*}}=\frac{1}{x^{*}}-\frac{\alpha}{1-x^{*}}
$$


or

$$
\mathrm{d} t^{*}=\frac{x^{*}-x^{* 2}}{1-(1+\alpha) x^{*}} \mathrm{~d} x^{*}=\left[\frac{1}{1+\alpha} x^{*}-\frac{\alpha}{(1+\alpha)^{2}}-\frac{\alpha}{(1+\alpha)^{2}} \frac{1}{(1+\alpha) x^{*}-1}\right] \mathrm{d} x^{*}
$$

With the initial condition $t^{*}(0)=0$ for any $x_{0}^{*}<1 /(1+\alpha)$, the filling time is determined as

$$
\begin{aligned}
t^{*}\left(x_{0}^{*}\right) & =\int_{0}^{x_{0}^{*}} \frac{1}{1+\alpha} x^{*} \mathrm{~d} x^{*}-\int_{0}^{x_{0}^{*}} \frac{\alpha}{(1+\alpha)^{2}} \mathrm{~d} x^{*}-\frac{\alpha}{(1+\alpha)^{2}} \int_{0}^{x_{0}^{*}} \frac{1}{(1+\alpha) x^{*}-1} \mathrm{~d} x^{*} \\
& =\frac{x_{0}^{* 2}}{2(1+\alpha)}-\frac{\alpha x_{0}^{*}}{(1+\alpha)^{2}}-\frac{\alpha}{(1+\alpha)^{3}} \ln \left[1-(1+\alpha) x_{0}^{*}\right]
\end{aligned}
$$

or

$$
t^{*}\left(x^{*}\right)=\frac{x^{* 2}}{2(1+\alpha)}-\frac{\alpha x^{*}}{(1+\alpha)^{2}}-\frac{\alpha}{(1+\alpha)^{3}} \ln \left[1-(1+\alpha) x^{*}\right]
$$

From Eq. (8), the final filling length is determined as

$$
\lim _{t^{*} \rightarrow \infty} x^{*}=\frac{1}{1+\alpha}
$$

Therefore, the filling length $x$ of the fluid cannot exceed $L /(1+\alpha)$. At this filling length, the force due to pressure of trapped gas volume is equal to the capillary force.

Considering the dissolution of gas in liquid, the mass of trapped gas in the channels reduces gradually over time. Under a high Laplace pressure, the gas dissolves in the liquid. The gas molecules in the liquid phase then diffuse out of the channels due to the gradient in concentration. The dissolution of gas into liquid phase is governed by Henry's law while the diffusion is governed by Fick's law. With the concentration $c$ of gas molecules at the meniscus, the Henry's law gives

$$
c=k_{\mathrm{H}} p
$$

The concentration of gas molecules at the entrance of the channel is therefore $c_{0}=k_{\mathrm{H}} p_{0}$. The 
Fick's law gives the mass flux of the gas molecules in the channel

$$
\Phi_{\mathrm{m}}=\frac{D_{\mathrm{d}} M\left(c-c_{0}\right)}{x}
$$

where $D_{\mathrm{d}}$ is the diffusion coefficient of the gas molecules in liquid and $M$ is the molar mass of the gas. The characteristic time for gas molecules to diffuse from one end to the other end of the nanochannel is $\tau_{\text {diffusion }}=L^{2} /\left(2 D_{\mathrm{d}}\right)$. The ideal gas law is applied to the trapped gas as:

$$
p=m \frac{R T}{M h w(L-x)}
$$

where $R$ is the universal gas constant and $T$ is absolute temperature. At $x=0$, the initial mass of gas is $m_{0}=p_{0} M h w L /(R T)$. The force balance Eq. (1) and the balance of the rate of mass change lead to the equation system:

$$
\left\{\begin{array}{l}
\frac{h}{12 \mu x}\left[2 \sigma \cos \theta-\frac{R T}{M w}\left(\frac{m}{L-x}-\frac{m_{0}}{L}\right)\right]=\frac{\mathrm{d} x}{\mathrm{~d} t} \\
\frac{D_{\mathrm{d}} R T k_{\mathrm{H}}}{x(L-x) L}\left[m L-m_{0}(L-x)\right]=-\frac{\mathrm{d} m}{\mathrm{~d} t}
\end{array}\right.
$$

Using the same dimensionless variables $t^{*}, x^{*}, \alpha$ and introducing the new dimensionless variables $m^{*}=m / m_{0}, \beta=\left(6 \mu D_{\mathrm{d}} R T k_{\mathrm{H}}\right) /(h \sigma \cos \theta)=R T k_{\mathrm{H}} \cdot \tau_{\text {filling }} / \tau_{\mathrm{diffusion}}$ make the equation system Eq. (13) dimensionless:

$$
\begin{cases}\frac{1}{x^{*}}-\alpha\left(\frac{m^{*}}{1-x^{*}}-1\right) & =\frac{\mathrm{d} x^{*}}{\mathrm{~d} t^{*}} \\ \beta \frac{1-m^{*}-x^{*}}{x^{*}\left(1-x^{*}\right)} & =\frac{\mathrm{d} m^{*}}{\mathrm{~d} t^{*}}\end{cases}
$$

The dimensionless number $\beta$ represents the relative ratio between the dissolving rate of the trapped gas and the filling rate of the liquid. Eq. (14) is difficult to be solved analytically, but can be solved numerically. Figure 4 compares the theoretical values predicted by the model with gas absorption [Eq. (14)] with those without gas absorption [Eq. (8)] for ethanol and isopropanol. Details on the values used for the theoretical curves and the experimental data are described in next section. 


\section{Experiments}

\section{Equipments and Materials}

The experiments were performed in nanochannels of $10 \mu \mathrm{m}$ width and $45 \mathrm{~nm}$ depth. The lengths of the nanochannels under investigation ranges from $0.5 \mathrm{~mm}$ to $2.5 \mathrm{~mm}$. The nanochannels were fabricated by reactive ion etching (RIE) process on a silicon wafer. The nanochannels were sealed by a glass wafer using anodic bonding. The entrance of the nanochannels are connected to a microchannel, which is accessible through reservoirs at both ends, Figure 1(b).

In the experiments, the silicon chip with the reservoirs facing up was observed using an inverted microscope (Zeiss Observer D1). The fluid is introduced into one of the reservoirs and starts to fill in the microchannel and nanochannels by capillary force. Ethanol, isopropanol and water were used as the filling fluids. To keep ethanol and isopropanol from wetting the outer surface of the chip, a spacer made from polydimethylsiloxane (PDMS) is placed above the reservoir. The temperature during all experiments was measured as $26 \pm 1{ }^{\circ} \mathrm{C}$. The relative humidity of the environment was $48 \%$. The images of the nanochannels were recorded by a CCD camera (EMCCD AndorIQ). The images were saved as a 16-bit gray-scale multi-images uncompressed TIF file with the size of 1004 pixels $\times 1002$ pixels.

\section{Methods}

The filling processes were observed with different magnifications and time scales, to determine the interaction among liquid, solid and air in closed-end nanochannels qualitatively and quantitatively. The experiments were carried out in three steps.

The first step records the filling process from the entrance until the end of the $3-\mathrm{mm}$ long nanochannels. Objective lens magnification of $2.5 \times$ were used. The field of view was approximately $3 \mathrm{~mm} \times 3 \mathrm{~mm}$. The exposure time was $10 \mathrm{~ms}$. The movie was recorded with a rate of 17 frames per second.

The second step records the development of the meniscus during the filling process. The closed 
ends of the 2-mm long nanochannels is chosen to observe this process. Objective lens magnification of $20 \times$ was used. Video setting was the same as in the first step.

The last step checks whether the liquid can fill until the end of the nanochannels after a long filling time. The still images of the closed ends are recorded with $20 \times$ magnification.

\section{Results and Discussions}

Figure 2 shows the square of the filling length versus the time of ethanol and isopropanol in nanochannels with different lengths. The linear characteristic of the curves at the beginning of the filling process suggests that the process follows Washburn's law in the early stage.

$$
x=\sqrt{a t}
$$

where $a$ is the so-called Washburn's coefficient and is $a=(\sigma \cos \theta h) /(3 \mu)$ for a slit-shape channel with a height of $h$. In Figure 2, the slopes of the data lines in neighbourhood of the axes origin are used to calculated the experimental Washburn's coefficients. The data of longest channel (2.5mm) in each case is used for numerical fitting because this channel length provides the most data points. Washburn's coefficient depends on the surface tension, the contact angle and the viscosity of the filling fluid. The contact angle for ethanol and iso-propanol is $\theta=0^{\circ}$. It is convenient to expressed the change in Washburn's coefficient using the effective viscosity and consider the surface tension and the contact angle unchanged from their bulk values. Using the experimental Washburn's coefficient, the effective viscosities of ethanol and isopropanol are taken as $177 \%$ and $195 \%$ of their respective bulk values at $25^{\circ} \mathrm{C}$.

In the model described in the previous section, we adopt isothermal hypothesis, which means the temperature of the system is stable and always equal to that of the environment. This assumption requires perfect heat dissipation. In practice, when the liquid advances in the nanochannel, the trapped air is compressed and increases temperature. The temperature of the compressed air is higher than that of the environment because of the finite heat transfer coefficients of materials. 

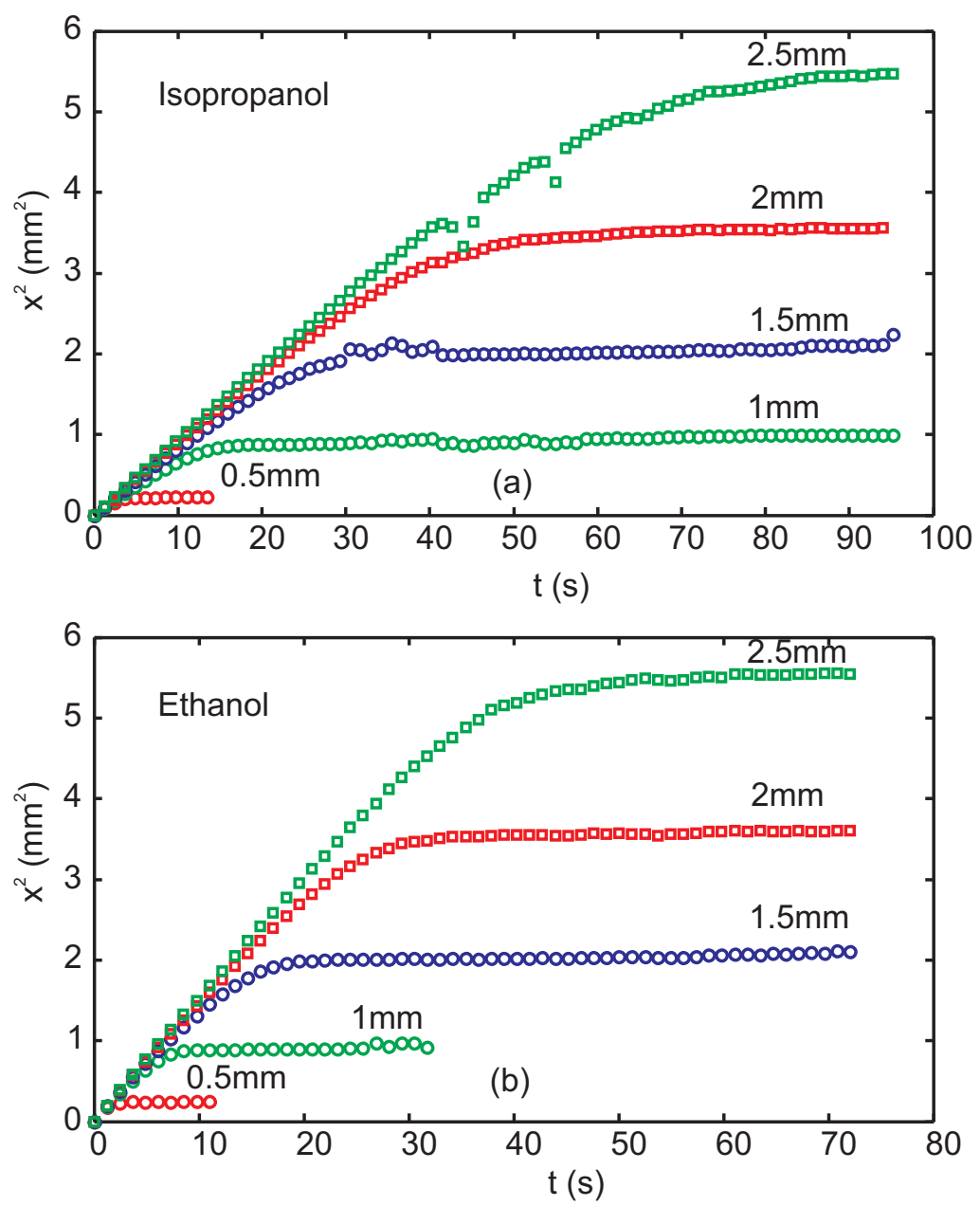

Figure 2: Filling length versus time in closed-end nanochannels with different length $L$ (a) Isopropanol ; (b) Ethanol. 
The pressure of the compressed air is therefore also higher than the value in our hypothesis. This additional pressure increasing further slows down the filling speed. For example, isopropanol fills in a 45-nm-height nanochannel leads to $\alpha \approx 0.1$, with $\alpha$ is the ratio between atmospheric ratio and Laplace pressure, as defined in Eq. (5). Compression ratio $r$ of the air is defined as the ratio between initial air volume and final air volume. With isothermal assumption, note that final pressure of the air should equal to the Laplace pressure:

$$
r=\frac{L}{L-x}=\frac{1}{1-x^{*}} .
$$

From Eq. (9), the compression ratio $r$ under isothermal assumption approaches a value of $(1+$ $\alpha) / \alpha \approx 10$, meaning $x \rightarrow 0.9 L$. Under an isentropic assumption (no heat dissipating) with a ratio of heat capacities $k \approx 1.4$, the pressure ratio is estimated as:

$$
\frac{1}{\alpha}=\frac{p_{\text {Laplace }}}{p_{0}}=\left(\frac{L}{L-x}\right)^{k}=\left(\frac{1}{1-x^{*}}\right)^{k} \approx 10
$$

The compression ratio approaches a value of $r=1 /\left(1-x^{*}\right) \approx 5$, meaning $x \rightarrow 0.8 L$. The limit of filling length with isentropic assumption is lower than that with isothermal assumption. This suggests that lower heat dissipation and increasing temperature may cause the reduction in filling speed, or the increase in effective viscosity. This phenomenon mainly affects the first filling stage, where a high compression ratio is reached in a short period of time. Hence, the isentropic condition could be assumed. However, analyzing the heat transfer process is complicated and beyond the scope of this article. Therefore, in this article, we used numerical fitting results for effective viscosity, although its value seem to be higher than those reported previously for the case of open channel. ${ }^{7-10}$

The same experiment was also performed using water as filling liquid. Qualitatively, the results show similar trend as those with ethanol and isopropanol. However, the chaotic region of the meniscus during second stage of filling is significantly larger as also observed previously by Pesse et al. ${ }^{11}$ The irregular meniscus and the droplet formation result in a large noise in the evaluated 
data, Figure 3. Images from the experiment using water were beyond our capability of image processing and data analysis. Therefore, this paper does not use water data to compare with the theory.

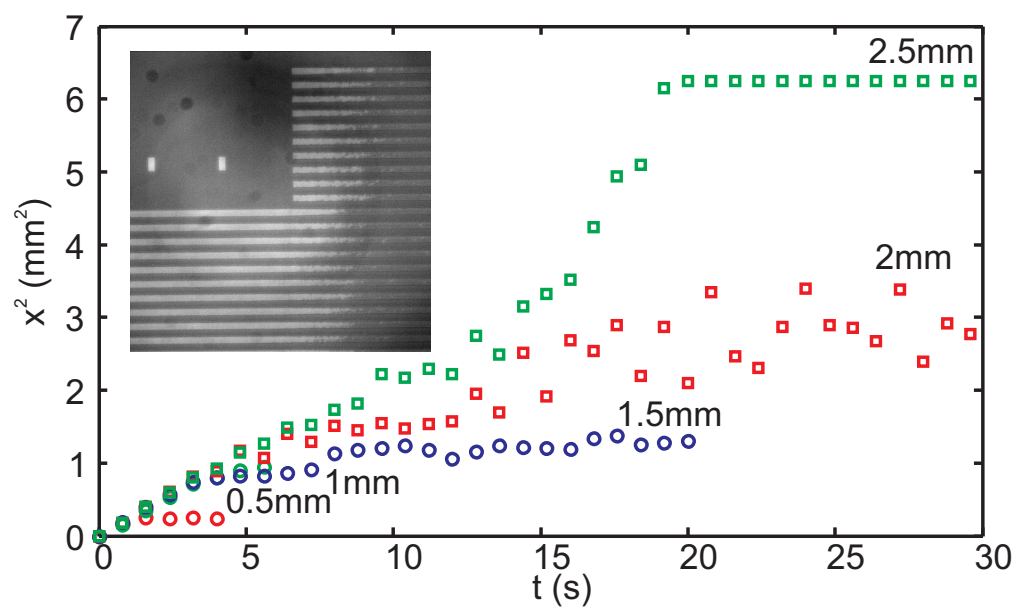

Figure 3: Filling length versus time of deionized water in closed-end nanochannels with different length $L$. The insert shows the end of $0.5 \mathrm{~mm}$ channels, with a large region of irregular meniscus, the main cause of noise in data analysis

Figure 4 shows the normalized filling length as a function of normalized time of the capillary filling process in closed-end nanochannels. Air is a mixture of oxygen, nitrogen and a minor amount of other gases. Due to the different Henry's coefficients and diffusion coefficients, a theory for such a mixture is more complex than that of a single gas as modeled by Eq. (14). In this work, we introduce effective values of air to serve the single-gas model. Few works in the past provided values of the Henry's coefficient $k_{\mathrm{H}}$ and the diffusion coefficient $D_{\mathrm{d}}$. Schnabel et al. used simulation method to predict the Henry's coefficient of various gases in ethanol, including oxygen and nitrogen. ${ }^{12}$ The simulation results were compared to previous experimental data. From this work, the Henry's coefficient of oxygen in ethanol at $300 \mathrm{~K}$ is approximately $9.5 \times 10^{-3} \mathrm{~mole} /(\mathrm{bar} \cdot \mathrm{liter})$ and that of nitrogen in ethanol is approximately $6.1 \times 10^{-3}$ mole $/($ bar $\cdot$ liter $)$. Ferrell and Himmelblau ${ }^{13}$ performed an experimental investigation on the diffusion coefficients of oxygen and nitrogen in water, which are $2.2 \times 10^{-9} \mathrm{~m}^{2} / \mathrm{s}$ and $2.0 \times 10^{-9} \mathrm{~m}^{2} / \mathrm{s}$ at $25^{\circ} \mathrm{C}$ respectively. These values are good approximations for the respective diffusion coefficients in ethanol and isopropanol. According to above results, the effective values of $k_{\mathrm{H}} D_{\mathrm{d}}$ in the case of air dissolve in ethanol and 
isopropanol are estimated to be on the order of $10^{-13}$ to $10^{-12} \mathrm{~mole} \cdot \mathrm{s} / \mathrm{kg}$. In Figure 4, values of $k_{\mathrm{H}} D_{\mathrm{d}}=6.5 \times 10^{-13} \mathrm{~mole} \cdot \mathrm{s} / \mathrm{kg}$ for ethanol and $3.5 \times 10^{-13} \mathrm{~mole} \cdot \mathrm{s} / \mathrm{kg}$ for isopropanol give good fitting to experimental data. The filling process consists of two stages. In the first stage, the force of the pressurized air $F_{\mathrm{P}}$ is negligible, the filling precess resembles that of capillary filling in openend nanochannels. In the second stage the trapped air is compressed under the effect of the surface force. The force $F_{\mathrm{P}}$ is comparable to the surface force $F_{\mathrm{S}}$. Under the high pressure, the absorption of air becomes more significant. The meniscus still slowly advances toward the end of the channel and finally fills the channel completely. This experimental observation agrees well with the model with gas absorption.

In the final filling state the shape of the meniscus is irregular. The chaotic movement of the meniscus create a region where both liquid and gas phases coexist, as shown by the inserts in Figures 3 and 5. This region causes the error in determination of the position of the meniscus. An indication for such errors is the scattered data of water shown in Figure 3, data of the 0.5-mm-long channel in Figure 4 and data of the second filling stage as shown in Figure 5(a). Therefore, to analyze this second stage, the remaining unfilled area of the nanochannels was measured. Images of the end of the nanochannels with a higher magnification were used for the analysis. Figure 5(a) shows the amount of unfilled area at the end of filling process of ethanol in a 2-mm-long nanochannel as a function of time. In Figure 5(a), the amount of unfilled area reduces linearly with time. This linear behavior is confirmed in Figure 5(b), which shows the changing rate of unfilled area as a function of time. The experimental results show that during the first stage of the filling process, the meniscus of the liquid advances faster and the shape of the meniscus is well defined. In the second stage, when the meniscus approaches the closed end, the filling speed is much slower and the shape of the meniscus becomes irregular. Two stages of filling are distinguished clearly in both cases of ethanol and isopropanol. During the second stage of filling, air bubbles are captured by irregular movement of the meniscus. The captured bubbles shrink and gradually disappear. This phenomenon can be considered as the dissolution process of air into the liquid under the high Laplace pressure. 

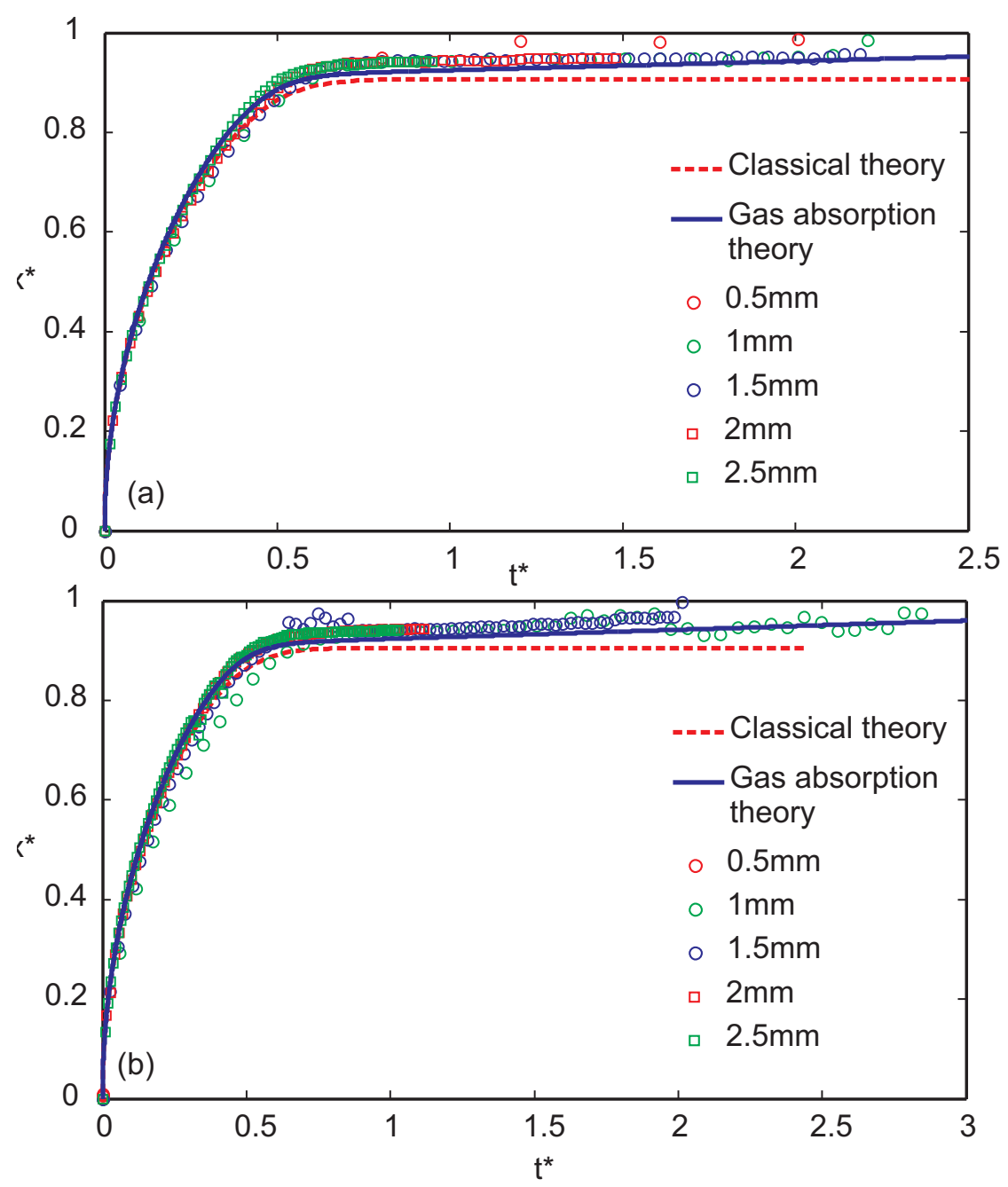

Figure 4: Normalized filling length versus normalized time in closed-end nanochannels with different length $L$ (solid line: model with gas absorption, dashed line: model without gas absorption, circles and squares: experimental data): (a) Isopropanol (effective viscosity is $177 \%$ of the bulk value); (b) Ethanol (effective viscosity is $195 \%$ of the bulk value). 

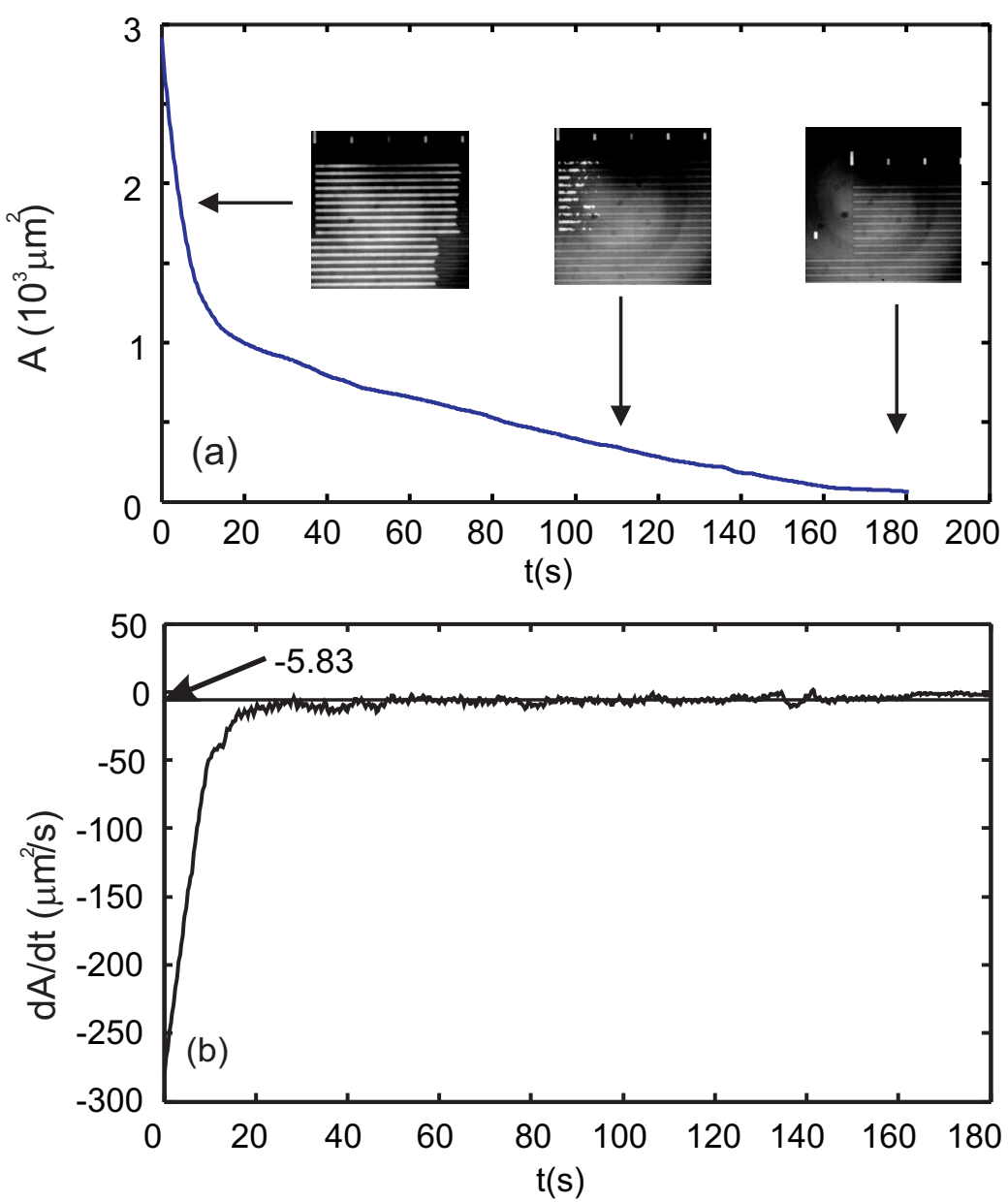

Figure 5: Unfilled area (a) and its changing rate (b) versus time at the end of filling process of Ethanol in a 2-mm-long nanochannel. 


\section{Conclusions}

This study investigates the capillary filling process of liquids in closed-end nanochannels. The results show that the process at first resembles the capillary filling process in open-end channels. However, at the end of the filling process, the menisci do not stop at the value predicted by the model without gas absorption, but continue to fill until the end of the nanochannels. The remaining gas trapped in the nanochannels dissolves in the liquid in the final stage of the filling process. A quantitative analysis of this stage shows a linear relationship between the filled area and the time. High magnification images reveal that air bubbles are captured by irregular movements of the air/liquid interfaces. Air bubbles reduce their size before totally disappear. This experimental result fit the model of absorption and diffusion of gas molecules in liquid. The phenomenon suggests that the dissolution of gas in the liquid is important in structure at nanoscale, where the Laplace pressure is high. The dissolution phenomenon of gas in liquid can influence the transport of fluid in nanochannels.

\section{References}

(1) Yiotis, A. G.; Stubos, A. K.; Boudouvis, A. G.; Yortsos, Y. C. Advances in Water Resources 2001, 24, 439-460.

(2) Prat, M. Chemical Engineering Journal 2002, 86, 153-164.

(3) Shaw, T. M. Physical Review Letters 1987, 59, 1671-1674.

(4) Prat, M. International Journal of Multiphase Flow 1993, 19, 691-704.

(5) Yiotis, A. G.; Boudouvis, A. G.; Stubos, A. K.; Tsimpanogiannis, I. N.; Yortsos, Y. C. Physical Review E - Statistical, Nonlinear, and Soft Matter Physics 2003, 68, 373031-373034.

(6) Eijkel, J. C. T.; Dan, B.; Reemeijer, H. W.; Hermes, D. C.; Bomer, J. G.; Van Den Berg, A. Physical Review Letters 2005, 95, 1-4. 
(7) Tas, N. R.; Haneveld, J.; Jansen, H. V.; Elwenspoek, M.; Van Den Berg, A. Applied Physics Letters 2004, 85, 3274-3276.

(8) Thamdrup, L. H.; Persson, F.; Bruus, H.; Kristensen, A.; Flyvbjerg, H. Applied Physics Letters 2007, 91, 163505-1-3.

(9) Han, A.; Mondin, G.; Hegelbach, N. G.; De Rooij, N. F.; Staufer, U. Journal of Colloid and Interface Science 2006, 293, 151-157.

(10) Phan, V. N.; Yang, C.; Nguyen, N. T. Microfluidics and Nanofluidics 2009, 1-12.

(11) Pesse, A. V.; Warrier, G. R.; Dhir, V. K. International Journal of Heat and Mass Transfer 2005, 48, 5150-5165.

(12) Schnabel, T.; Vrabec, J.; Hasse, H. Fluid Phase Equilibria 2005, 233, 134-143.

(13) Ferrell, R. T.; Himmelblau, D. M. Journal of Chemical and Engineering Data 1967, 12, $111-115$. 